\title{
Model for dynamics of structural glasses
}

\author{
Ian S. Graham, ${ }^{1}$ Luc Piché, ${ }^{2}$ and Martin Grant ${ }^{3}$ \\ ${ }^{1}$ Instructional and Research Computing, Computing and Communications, 4 Bancroft Avenue, University of Toronto, \\ Toronto, Ontario, Canada M5S 1A1 \\ ${ }^{2}$ National Research Council of Canada, Industrial Materials Institute, 75 De Mortagne Boulevard, \\ Boucherville, Québec, Canada J4B $6 Y 4$ \\ ${ }^{3}$ Physics Department, McGill University, Rutherford Building, 3600 rue University, Montréal, Québec, Canada H3A 2T8
}

(Received 7 July 1995; revised manuscript received 28 May 1996)

\begin{abstract}
We have studied, using dynamical Monte Carlo methods, a facilitated kinetic Ising model for structural glasses. We observe stretched-exponential decays ( $\alpha$ relaxation) of the equilibrium spin autocorrelation function at late times and are able to estimate accurately the corresponding relaxation times $\tau$. These are found to diverge at nonzero temperatures following a Vogel-Fulcher law. In addition, we observe early-time exponential relaxation analogous to $\beta$ relaxation of glass formers. We also examined the effective thermodynamics of systems quenched below this divergent temperature and subsequently heated. The result is a peak in the specific heat with properties matching those of the putative glass transition. We also find that these peaks, for different heating rates, can be rescaled to lie on a universal curve. Finally, we studied the evolving structure factor of a frozen glass following a rapid jump in temperature. The results are qualitatively the same as those for x-ray studies of heated glasses. We thus demonstrate that this purely dynamical microscopic model can reproduce much of the phenomenology of the glass transition and near-glass relaxation, with no tunable parameters. [S1063-651X(97)07301-7]
\end{abstract}

PACS number(s): 05.50. $+\mathrm{q}, 43.20 .+\mathrm{g}, 63.20 . \mathrm{Pw}, 71.55 . \mathrm{Jv}$

\section{INTRODUCTION}

Recently, there has been renewed experimental and theoretical interest in structural glasses and the glass transition. New experimental methods have allowed for detailed study of the dynamical processes taking place in highly viscous fluids. At the same time new theoretical approaches, in particular mode-coupling theories, have provided explanations for some of these observations, in particular in the mediumto high-viscosity region above the experimental glass transition. However, the nature of the highly viscous fluid near the glass, and the glass transition itself, remains unresolved [1-4].

Unlike a spin glass, the Hamiltonian of glass-forming materials contains no intrinsic disorder. Instead the structural disorder arises from a dynamical mechanism (rapid cooling, for example) that keeps the system from forming an ordered equilibrium phase. It is currently believed that the resulting structural glass may be not a proper equilibrium phase but instead a material trapped in a long-lived metastable state. However, the dynamical mechanism causing this trapping is unclear, as is how such a mechanism can lead to the observed experimental phenomenology. For example, given a mechanism we would like to know how its onset, as one approaches the glass transition temperature $T_{g}$, gives rise to nonexponential decays of the autocorrelation or relaxation functions and to an apparent divergence in the relaxation time at a nonzero temperature $T_{0}$ below $T_{g}$, often characterized by the Vogel-Fulcher law $\tau \sim e^{A / k\left(T-T_{0}\right)}$ [5], where $A$ is a constant energy scale and $k$ is Boltzmann's constant. We also would like to know how the mechanism can yield thermal properties of the glass transition that mimic closely those of a true thermodynamic phase transition. Finally, we would like to know if the transition to a glass represents a new class of true dynamical transitions. Given these questions, much effort has been devoted to developing theories and models for glasses and glassy relaxation. To date, however, there is no satisfactory description relating a microscopic mechanism for slowed relaxation to the relaxation time divergence near the glass transition or to the glass transition itself. Finding such a mechanism would be of great use in the development of a rigorous theory for glasses.

In this paper we test the idea that dynamical frustration is the relevant mechanism. We do this using a microscopic dynamical spin model for glasses and glassy relaxation. The starting point is a kinetic Ising model in the absence of spinspin interactions $(J=0)$. By itself this model is trivial: with $J=0$ there is no thermodynamic transition at any temperature. All relaxation and correlation functions decay exponentially, in or out of equilibrium, and all spins are uncorrelated. We introduce dynamical frustration by adding a dynamical rule for frustration to the Monte Carlo dynamics. This rule, defined below, satisfies detailed balance, so that the eventual equilibrium state remains unchanged, but the dynamical process leading to equilibrium changes markedly. Such an idea was proposed in the facilitated kinetic Ising model of Fredrickson and Andersen [6]. Their analytic study predicted that their model should have a power-law singularity in the equilibrium relaxation time at nonzero temperature, which they interpreted as a dynamical transition leading to a glass, although subsequent simulation studies did not support the existence of this power-law singularity $[7,8]$.

Notwithstanding the absence of the power-law singularity, we have revisited this model and made a large-scale numerical study. This is because we believe its dynamical frustration can provide the most simple representation of glassy behavior. We have used a simplified version of the facilitation model, which we studied in both two and three 
dimensions and for several facilitation rules. We examined the properties of the model for temperatures where equilibrium was possible and also the effective thermodynamics upon heating from and cooling into the essentially glassy state. Our results are as follows. At low temperatures the equilibrium autocorrelation functions have the late-time stretched exponential decays characteristic of glass-forming materials. More importantly, the relaxation time $\tau$ that we extract from these data diverges at nonzero temperatures following a Vogel-Fulcher law. In addition, our simulations of heating and cooling of a quenched low-temperature "glass" show a peak in the effective specific heat with properties very similar to those of the glass transition. We also find that data for different heating rates can be scaled onto a universal curve. These results are independent of the dimensionality of the model or of the facilitation rules employed. Finally, our structural study of rapidly heated glasses shows behavior similar to that seen in dynamical $x$-ray studies of rapidly heated glasses. Thus this simple model is able to reproduce much of the phenomenology of glassy relaxation and the glass transition, with no adjustable parameters [9].

The paper is organized as follows. In the next section we review the experimental phenomenology of glasses along with the results of molecular-dynamics simulations of model systems. Following this is a review of some models and theories. We next introduce our model, discussing its implementation and theoretical underpinnings. Finally, we present our results and discuss them in the context of current experimental measurements on glasses.

\section{REVIEW}

Glasses have been extensively studied over the past half century, so that the basic phenomenology is well known and has been extensively reviewed [1-4]. Here we summarize these general results. We discuss in more detail experiments results on dynamics in glassy materials, as well as the status of current theory.

A qualitative understanding of the glass transition can be obtained from the free-volume approach $[10,11]$. This model predicts a Vogel-Fulcher form for the relaxation time by postulating a simple relationship between the free volume available for structural relaxation and the corresponding viscosity or relaxation time. By assuming a minimum possible free volume $v_{0}$ (e.g., analogous to the free volume of a random close-packed system of hard spheres) one argues that the relaxation time will diverge $\sim e^{O\left(1 /\left(v_{f}-v_{0}\right)\right)}$, where $v_{f}$ is the actual free volume available [12]. Linearizing $v_{f}(T)$ yields the Vogel-Fulcher form. Of course, such an approach does not take into account the nonequilibrium nature of the transition. Nevertheless, the key idea of this phenomenological approach, that relaxation on long length scales is frustrated by the local structure (represented here by an average quantity, the free volume), is appealing and appears in one form or another in most modern approaches.

Subtleties with the glass transition begin with defining a precise temperature at which it occurs. The glass transition temperature $T_{g}$ can be operationally defined as the point upon cooling where the measured viscosity exceeds $\eta \approx 10^{13} \mathrm{P}$. Alternatively, one can heat a frozen glass and examine the changing specific heat. In this case the glass transition is defined by the onset temperature at which, in the absence of crystallization, the specific heat shows a prominent peak or jump [13]. One can also measure changing ultrasonic acoustic properties as a glass is heated, in which case the glass transition is indicated by a kink in a plot of acoustic attenuation (or velocity) as a function of temperature $[14,15]$. Finally, one can measure the sample specific volume as a function of temperature [16]. This curve shows a kink at a temperature consistent with the $T_{g}$ predicted by acoustic attenuation or specific heat measurements. Therefore, provided the measurement time scale $(\mathrm{MHz}$ for the acoustic studies, effectively $\mathrm{mHz}$ for viscoelastic properties, zero $\mathrm{Hz}$ for specific volume) is smaller than the experimental time scale from the scanning rate $[d(\ln T) / d t]^{-1}$, the glass transition temperature appears to be frequency independent.

The actual value of $T_{g}$ depends on the time scale of the experiment, i.e., heating or cooling rates, and on the sample history (annealing time at low temperature, for example) consistent with the idea that the transition is a nonequilibrium phenomenon. An experimental study of the heating-rate dependence of $T_{g}$ and of the width of the specific-heat peak has been undertaken, with the aim of determining if $T_{g}$ and the heating rate are related by a Vogel-Fulcher law and if the transition width extrapolates to zero at a finite heating rate [17], with as-yet inconclusive results.

As one approaches $T_{g}$ from higher temperatures, glassforming materials show complex nonexponential decays in autocorrelation or relaxation functions. The time constants $\tau$ involved in these dynamic processes are strongly temperature dependent. The dependence of $\tau(T)$ can often be extrapolated to show apparent divergences at nonzero temperatures. Viscoelastic [18,19], light scattering [20-22], NMR $[18,23,24]$, ultrasonic [15], specific-heat spectroscopy [2527], dielectric response [28-30], and neutron-scattering [3138] studies of highly viscous glass formers have shown complex relaxational behavior. This is also evident in moleculardynamics simulations $[39,40]$ of model systems that have shown that the relaxation process is quite complex. Early dielectric relaxation studies [30] of supercooled fluids indicated two distinct mechanisms, now known as $\alpha$ and $\beta$ relaxation. This was later confirmed in neutron-scattering $[34,36,38]$ studies and by molecular-dynamics simulations $[41,42]$.

Loosely speaking, $\alpha$ relaxation is associated with longtime scales and long-range order at the glass transition, while the faster $\beta$ relaxation involves shorter time scales and is associated with short-range order. Indeed, the transient process, $\beta$ relaxation, appears at temperatures above $T_{g}$ and persists into the glass. Through study of ionic and polymeric glasses above the glass transition this mechanism has been observed to decay via power laws with temperaturedependent exponents $[20,34]$. Depending on the time scales considered, the corresponding relaxation times appear to have an Arrhenius temperature dependence $\left(e^{A / k T}\right.$, where $A$ is an activation energy and $k$ is Boltzmann's constant) or for somewhat longer times an effective power-law form. As temperatures are reduced (well above $T_{g}$ ) the temperature dependence of short-time $\beta$ relaxation follows an effective power-law dependence of the viscosity $\eta \sim\left(T-T^{\prime}\right)^{-\mu}$, with $T^{\prime}>T_{g}$ and $\mu \sim 2$ [43]. The form is only an effective one, since as temperatures are reduced towards $T^{\prime}, \alpha$ relaxation, 
which involves Vogel-Fulcher temperature dependences, dominates $\beta$ relaxation. The temperature $T^{\prime}$ marks a crossover boundary between two different regions of viscous behavior [43]. This crossover occurs at a viscosity of around $10^{2}-10^{3} \mathrm{P}$, a region readily accessible to other experimental techniques and to molecular-dynamics simulations. In fact, experimental results have been obtained in neutronscattering studies [36,38], while the two distinct $\alpha$ and $\beta$ mechanisms have also been observed in molecular-dynamics simulations $[41,42]$. These experimental results on $\beta$ relaxation have been taken as confirmation of mode-coupling theories, as discussed in two reviews [44,45].

The long-time mechanism, $\alpha$ relaxation, is observed only at temperatures above $T_{g}$. As the temperature is lowered towards $T_{g}$ this feature dominates the late-time behavior and is characterized by nonexponential relaxation and divergence of the relaxation times near the glass transition temperature. It is the $\alpha$ mechanism that is most directly associated with long-range order at the glass transition. At late times this decay is often well described by a stretched-exponential (also known as a Kohlrausch) decay

$$
\Phi(t) \sim e^{-(t / \tau)^{\alpha}}
$$

with $\alpha<1$, where $\Phi(t)$ is a correlation function defined below. The stretch exponent $\alpha$ does not appear to be universal and can vary from 0.2 to 0.8 depending on the material being studied, the method of sample preparation, and in some cases the temperature. The appropriateness of this expression has been questioned [29] and other functions have been suggested, particularly in the context of dielectric response measurements [46]. However, the stretch form is widely used due to its reliability in reproducing data from many different experiments and as a general scaling form. We reiterate, though, that such a function is phenomenological.

As temperature is lowered and the glass transition approached the relaxation time $\tau$ or viscosity grows rapidly and in many cases extrapolates to a divergence at a temperature $T_{0}<T_{g}$. This divergence is often well fit by the expression

$$
\tau \sim e^{A /\left[k\left(T-T_{0}\right)\right]},
$$

known as the Vogel-Fulcher law when $T_{0}>0$. Some materials are best described with $T_{0}=0$, i.e., Arrhenius relaxation with activation energy $A$. Such materials typically form strong covalently bonded networks, for example silicates. Most glass-forming materials, such as polymers, metallic glasses, and ionic systems do not form strong covalent bonds or bonded networks. These materials show non-Arrhenius relaxation near the glass transition, characterized by an apparent divergence at a nonzero temperature $T_{0}<T_{g}$.

One question is whether there is a simple and universal form for $\alpha$ relaxation. Neutron spin-echo experiments suggest that near $T_{g}$ the Kohlrausch decay $c e^{-(t / \tau)^{\alpha}}$ may satisfy this requirement with a rescaling of times $t / \tau(T)[34,37]$. These data also suggest that the prefactor $c$ is temperature independent well above $T_{g}$, but that it acquires temperature dependence close to the transition (a nondiverging power law). These results are consistent with several moleculardynamics simulations $[41,42,47,48]$, but not all [49]. Meanwhile, the dielectric relaxation studies of Dixon et al. [25,29] suggest a more complicated universal form similar to that seen in multifractal scaling: There the Kohlrausch form does not appear to reproduce the high-frequency data. Other results suggest that Dixon and co-workers' scaling is not universal and has to be modified to account for high-molecularweight polymers [28]. The discrepancy between the dielectric and neutron-scattering experiments has yet to be resolved.

We can summarize as follows. Near the glass transition, autocorrelation functions decay nonexponentially at late times by $\alpha$ relaxation, with the form often being well described by a stretched exponential. The corresponding relaxation times usually extrapolate to a divergence, via the Vogel-Fulcher law, at a nonzero temperature below that of the glass transition. Network glasses show the more straightforward Arrhenius form. There are also faster transient $\beta$-relaxation mechanisms that decay via power laws and whose relaxation times appear to diverge via Arrhenius laws, although when glass formers are studied at temperatures well above the glass transition these mechanisms suggest effective power-law divergence of the viscosity at a temperature higher than the glass transition. A critical examination of models of the temperature dependence of glass-forming liquids has been given recently by Stickel et al. [50].

Theoretical investigations of glassy phenomena have followed several approaches with mixed success. These include thermodynamic free-volume models [10], blocking or diffusion models [51], hierarchical models [52], and hydrodynamic mode-coupling theories [44,45,53-57]. The oldest is the free-volume approach discussed above. This idea of local structure trapping the system in a metastable state has been tested by several constrained dynamics models $[3,51]$ and via hierarchical relaxation models [52].

The former approach constructs an artificial dynamics that serves to slow relaxation. The results often yield nonexponential relaxation functions and in some cases a nonArrhenius divergence of the relaxation time. However, in general, the models are too artificial to be easily related to real systems. An exception is the facilitation kinetic Ising model. This model, introduced by Fredrickson and Andersen $[6,7]$, attempted to surmount these problems by incorporating dynamical frustrated relaxation into a simple microscopic model: the kinetic Ising model. Dynamical frustration is incorporated by allowing a spin to relax only when the neighboring spins are in an appropriate state, analogous to allowing a flip only when a site is surrounded by sufficient local free volume. Analytic study [6] of one version of this model suggested power-law divergence of the relaxation time at a nonzero temperature and stretched-exponential decays for the autocorrelation functions. Subsequent numerical work in two dimensions verified stretched-exponential decay of the autocorrelation function, but found only weak evidence for divergence of the relaxation time at nonzero temperatures. Hierarchical models complement these constrained dynamics approaches. The model proposes a hierarchy of relaxation processes such that a state at a high level, corresponding to a long relaxation time, can relax only if a certain set of states on lower hierarchy levels (with shorter relaxation time) have also relaxed. This approach can yield Vogel-Fulcher laws and stretch-exponential decays, given appropriate assumptions about the relationship between the levels of the hierar- 
chy. In fact, this approach has been used successfully to describe experimental data on relaxation in ionic glasses [58]. However, it is difficult to relate the model to the underlying physics, as there is no obvious prescription for determining the rules of the hierarchy from the microscopic properties.

The above approaches are similar in that they consider glassy phenomena to arise from frustration arising from the local structure. A conceptually different approach is hydrodynamic mode-coupling theory $[44,45,53-57]$. This theory has met with some success, particularly in the moderately viscous region prior to the formation of a glass. The essential idea is that a glass arises from a hydrodynamic slowing down near the glass transition, where the slowing down is governed by a nonlinear coupling between the density fluctuations and the renormalized viscosity. The resulting model predicts several different scaling regimes, two of which were mentioned above in the context of neutron-scattering results. These neutron results have been interpreted as a confirmation of the predictions of mode-coupling theory. However, the agreement with experiment requires terms within the memory kernel linking the density fluctuations and the renormalized viscosity that are difficult to justify theoretically [45]. As a result, any hydrodynamic mechanisms leading to glassy relaxation and the glass transition are obscured.

\section{MODEL}

The problem in simulating a glass is one of time, as the time scale of atomic motion is many orders of magnitude shorter than the time scale on which glassy phenomena are observed. Thus, for example, molecular-dynamics calculations are limited to the moderately viscous regime $\eta \approx 10^{3} \mathrm{P}$, where precursor effects to glasses may be observed, but are far from the glassy regime ten orders of magnitude away. Monte Carlo methods are similarly, though less restricted. Our approach is slightly different: we postulate a dynamical mechanism that gives rise to frustrated relaxation and glassy behavior and incorporate it directly into the dynamics of the model. As a result, the effective time scale of our simulations is on the scale of the frustration and not on the shorter time scale of atomic motion. We can therefore simulate much later effective times and can test, by our results, whether or not our postulated mechanism is correct.

A reasonable candidate for a mechanism are local barriers to relaxation that depend on the local environment. Such barriers will inhibit relaxation when the neighborhood is too tightly packed, in keeping with the frustration ideas discussed above. In general, the height of these barriers may depend on the local environment. To simplify the model we assume only two possible barrier energies: zero or infinity. This should retain the essence of the model while preserving the universal behavior.

We test this idea within a variant of the facilitation model of Fredrickson and Andersen, in both two and three dimensions. Following them, we consider a kinetic Ising model in the absence of any site-site coupling $(J=0)$, but in the presence of an external field. For convenience we set the external field to unity (or, equivalently, temperature is measured in units of the strength of the external field), defining a positive field as being in the negative spin direction. The Hamiltonian is

$$
\mathcal{H}=\sum_{i=1}^{N} \sigma_{i}
$$

where $\sigma_{i}= \pm 1$ is the spin at site $i$ and $N$ is the number of spins. There is no underlying thermodynamic phase transition; the system is always paramagnetic. In the absence of any frustration rules the equilibrium and nonequilibrium properties on the model are well known. All equilibrium or nonequilibrium autocorrelation functions decay exponentially in time and the average spin at equilibrium is $m_{0}=\langle\sigma\rangle=-\tanh (1 / k T)$. In addition, the spins are spatially uncorrelated, at all times, whether or not the system is at equilibrium.

To add dynamical frustration, denoted facilitation, we add a rule such that a spin can attempt a flip (with standard Boltzmann probability) only if the spin is surrounded by a sufficient number of up spins. Allowing the attempted flip is equivalent to saying there is no barrier to the transition, while forbidding the flip implies an infinite barrier height. For any lattice there can be several facilitation rules. A twosite facilitation rule is one where a spin flip is allowed only if two or more of the nearest-neighbor spins are in the spin-up state. Thus the three-site rule requires a minimum of three up-spin neighbors, and so on. Such rules clearly serve to slow the dynamics, and we can anticipate that the harder the rule, the slower the evolution.

The kinetics of this model are easily implemented within Metropolis Monte Carlo dynamics by adding the facilitation rule to the transition probabilities. They become

$$
P_{a \rightarrow b}=f\left(\left\{\sigma_{j}\right\}\right) \times\left\{\begin{array}{l}
e^{-\left(E_{a}-E_{b}\right) / k T} \text { for } E_{a}>E_{b} \\
1 \text { for } E_{a} \leqslant E_{b},
\end{array}\right.
$$

where $E_{a}=-\sigma_{i}$ and $E_{b}=\sigma_{i}$ are the energies at site $i$ (initially with spin $\sigma_{i}$ ) before and after the attempted flip, respectively. Time is measured in units of Monte Carlo steps per spin (MCS/spin). Each Monte Carlo step corresponds to $N$ attempts to change the system's state using this transition probablitity. The facilitation function $f\left(\left\{\sigma_{j}\right\}\right)$ depends only on the states of the spins $\left\{\sigma_{j}\right\}$ neighboring the site of interest and is either 1 or 0 depending on whether or not there are sufficient neighbors to satisfy the facilitation rule [59]. (Consequently, distances are measured in units of the distance between neighboring sites.) The effect of this rule is to slow the dynamics in such a way that the equilibrium thermal averages are preserved. The glassy behavior we investigate here is associated with this slow dynamics. This point has been discussed elsewhere [7].

The facilitation model was incorporated within a multispin algorithm previously used to study equilibrium and nonequilibrium properties of the kinetic Ising model [60]. This allows us to simulate 32 independent systems in parallel. In two dimensions we examined two-site facilitation, while in three dimensions we studied both two-site and three-site models. The advantage of our algorithm is its speed and ability to average over many independent systems. This is important, as the phenomena we wish to study are at late times, where good statistics are necessary to extract data from the background. Using our algorithm we are able to 
produce results to later times and with much higher precision that has heretofore been possible.

Three different initial conditions were considered for our numerical studies: (i) we prepared a system initially with all spins up, quenched the system by applying a reversed field, and monitored the subsequent evolution; (ii) we prepared the system in a magnetized equilibrium state and estimated the time-dependent fluctuations around this state; and (iii) we examined the thermal fluctuating properties of glassy state, which was prepared by quenching below the critical relaxation temperature (discussed below) and annealing the system for several $10^{6} \mathrm{MCS} / \mathrm{spin}$.

For the system quenched by the reversed fixed external field $1 / k T$, we monitored the evolution of the average magnetization $m(t)=\langle\sigma\rangle$ as the system evolved to equilibrium. At late times this magnetization reaches the equilibrium value $m_{\infty}=\lim _{t \rightarrow \infty} m(t)$. For sufficiently large systems $m_{\infty} \equiv m_{0}$, so that the equilibrium average is equal to that predicted by the equilibrium partition function. As noted previously [7], facilitation can trap the system within a restricted configuration space, provided the quench field is too deep for a given system size. In this case $m_{\infty}>m_{0}$. We used this criterion to detect finite-size effects in our simulations and have accordingly increased system sizes to give correct results. Provided $m_{\infty} \equiv m_{0}$, to within statistical error, we find that other quantities we measure, in particular the relaxation times, are independent of the size of the system. For dimension $d=2$, we used systems of size $N=24^{2}-48^{2}$, while for $d=3$, we used $N=8^{3}-16^{3}$. Magnetizations $m(t)$ were averaged over anywhere from 640 to several thousand independent quenches depending on the time required for the system to actually equilibrate. We then calculated the normalized relaxation function

$$
\psi(t)=\frac{m(t)-m_{\infty}}{1-m_{\infty}} .
$$

In some cases we also calculated the spatial correlation function $g(r, t)$ during the quench and at equilibrium $(t \rightarrow \infty)$. This is defined by the average

$$
g(r, t)=\left\langle\frac{\langle\sigma(r) \sigma(0)\rangle-m(t)^{2}}{1-m(t)^{2}}\right\rangle
$$

where the inner averages $\langle\sigma(r) \sigma(0)\rangle$ and $m(t)$ are taken over all appropriate spins for a given system and the outer average is an ensemble average. In the absence of facilitation, $g(r, t)=\delta_{r=0}$, since all sites are uncorrelated. This should also be the case in the presence of the facilitation, but only at equilibrium, since facilitation should affect the nonequilibrium and not the equilibrium properties of the system. We examined equivalently the radially averaged power spectrum of the lattices $S(q, t)$, which is the Fourier transform of $g(r, t)$, suitably normalized in real space to give $S(0, t) \equiv 0$. A purely uncorrelated system yields a flat power spectrum, with the amplitude reflecting the noise strength (magnitude of the fluctuations).

For the systems prepared in equilibrium, we also measured the spin-spin autocorrelation function. In normalized form this is

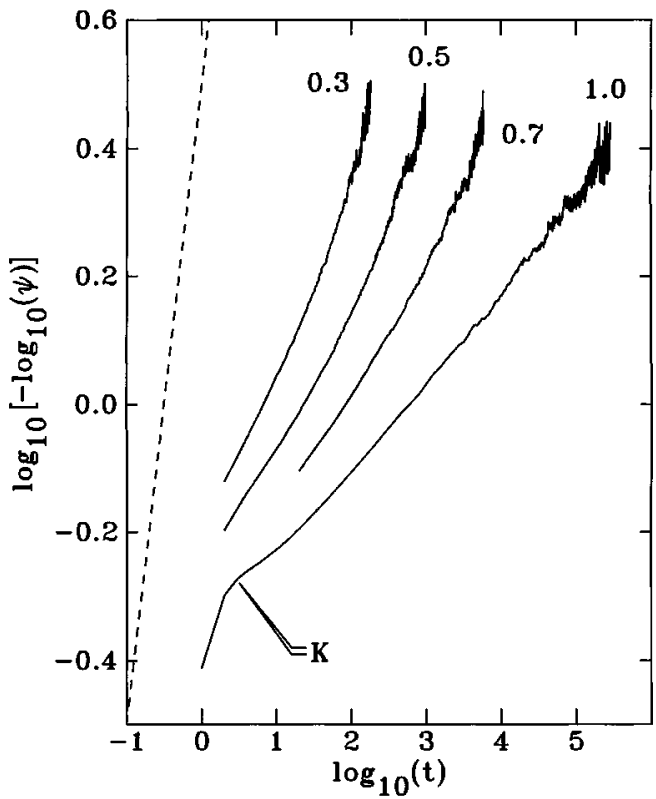

FIG. 1. $\log -\log$ plot of $-\log _{10} \psi(t)$, as a function of time $t$ following a quench from the all spin-up state, where $\psi(t)$ is the relaxation function. The results here are for a two-dimensional system, using the two-site facilitation rule, at differing values of $1 / k T$ as indicated. The results for other dimensions and models are qualitatively the same. The arrow $K$ marks the kink in the relaxation function, as discussed in the text. The dashed line corresponds to ordinary exponential decay $\alpha=1$.

$$
\Phi(t)=\frac{\left\langle\sigma_{i}(t) \sigma_{i}(0)\right\rangle-m_{\infty}^{2}}{1-m_{\infty}^{2}}
$$

where the average \langle\rangle is over all sites. The averages were again taken over from 640 to several thousand independent runs. From $\Phi(t)$, we obtain a characteristic time scale $\tau(T)$ from the integral of the correlation function $\int_{0}^{\infty} \Phi(t) d t$ or by simply fitting $\Phi(t)$ to a form.

Finally, the prepared glassy state was studied in two ways. We first considered slow uniform heating of the system and measured during this process the effective specific heat as a function of temperature $T$. This is analogous to a calorimetric measurement of the glass transition. Second we considered the response of the system to a temperature jump to higher temperature, monitoring in this case the average magnetization $m(t)$ and the structure factor $S(q, t)$. This is of interest with reference to a time-resolved $x$-ray study of amorphous metals [61], which examined the early-time evolution of the structure factor.

\section{RESULTS}

Typical results from quenching with the external reversing field are shown in Fig. 1 for different inverse temperatures $1 / k T$. These results are qualitatively independent of the dimensionality or of the choice of facilitation models: the only apparent effect of making the facilitation more restrictive is to slow the dynamics. There are two clear regions in these data: an early-time exponential, followed by late-time slower-than-exponential decay. The crossover from early to late time is interesting: for a short time evolution slows sig- 


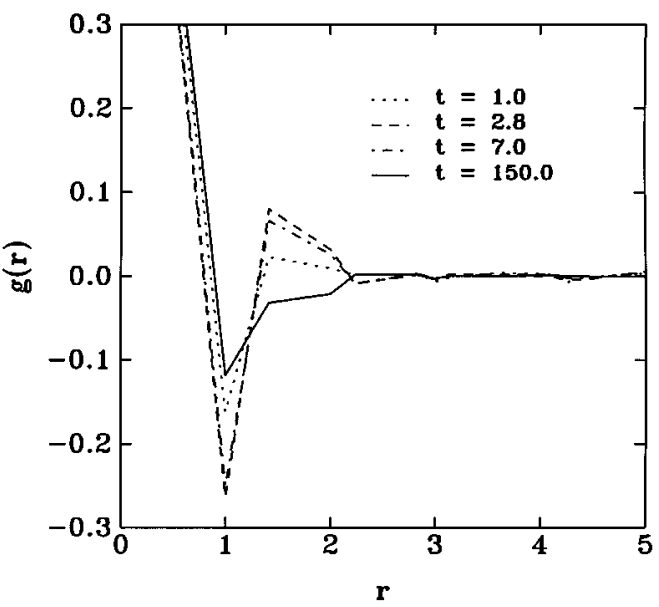

FIG. 2. Pair correlation function $g(r)$ plotted for different times $t$ following a quench from the all spin-up state. The buildup of correlations at intermediate times is clearly evident. The maximum amplitude of these correlations occurs at a time coincident with the kink marked in Fig. 1.

nificantly, after which the decay begins again, but slower than exponential. This behavior, indicated by the arrow $K$, can be explained as follows. At early times most of the spins are up, so it is easy to flip them down. Here the facilitation rule plays almost no role, so decay is exponential. Eventually all the easy spins have been flipped and the remaining up spins cannot flip until their environment has been arranged to bring up spins into their neighborhood. This initial phase of up-spin diffusion is associated with very little change in the average magnetization, since motion of spins requires spins to be flipped up and then down: no net change in magnetization occurs. Therefore the decay in $m(t)$ slows significantly. Eventually, however, clusters of up spins form and allow the decay to continue, albeit slower than exponentially.

Evidence of this is seen in the dynamical evolution of the pair correlation function, shown in Fig. 2. At early and late times the spins are uncorrelated, as expected, but at intermediate times we see the buildup of short-range correlations, which dissipate as equilibrium is approached. The maximum in this anomalous correlation takes place at the time when the dynamical evolution is slowest. These correlations have a purely dynamical origin and disappear at late times as the equilibrium state is achieved. Thus the facilitation rule does not introduce equilibrium correlations on any length scale, as expected.

We now turn to the behavior at equilibrium. In Fig. 3 we plot characteristic results for the equilibrium autocorrelation functions, shown here for a three-dimensional system with the two-site facilitation rule. At low temperatures (high fields) the late-time behavior is well described by a stretch exponential, with the stretch slope $\alpha$ decreasing slightly with decreasing temperature. This is $\alpha$ relaxation in the model. We fit the late-time data to the function $e^{-(t / \tau)^{\alpha}}$ to determine the stretch power $\alpha$ and relaxation time $\tau$. To test the appropriateness of this form we plotted $\ln (\Phi)$ as a function of $\ln (t)$ : there was no evidence for late-time power-law behavior. We also fit the late-time data to the form $c e^{-(t / \tau)^{\alpha}}$, as well as to the sum of ordinary exponentials $c e^{-t / \tau_{1}}+(1-c) e^{-t / \tau_{2}}$. In the former case, the best-fit esti-

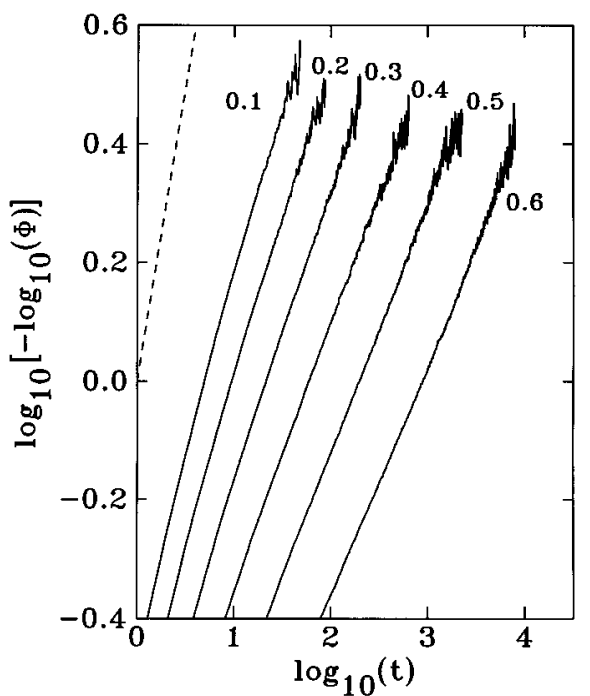

FIG. 3. $\log -\log$ plot of $-\log _{10} \Phi(t)$, as a function of time $t$ for temperatures, from left to right, of $1 / k T=0.1,0.2,0.3,0.4,0.5$, and 0.6, where $\Psi(t)$ is the autocorrelation function. The results here are for a three-dimensional system, using the three-site facilitation rule. Straight lines correspond to a stretched exponential, the slope being the stretch power $\alpha$. The dashed line corresponds to ordinary exponential decay $\alpha=1$.

mates were most consistent with $c \simeq 1$, there being no systematic variation of $c$ with temperature. This is different from hydrodynamic mode-coupling theory, which predicts a cusplike temperature dependence in this quantity. Comparing the fits, we found that the stretch form represented the data better than the sum of exponentials. We can also estimate the relaxation time $\tau$ from the integrated autocorrelation function. This gives an estimate entirely consistent with that obtained from the fits, implying that the integral of $\Phi(t)$ is dominated by a stretched exponential.

For times shorter than those shown in Fig. 3 the decays of the autocorrelation function are exponential. In the model, this time regime is what corresponds to $\beta$ relaxation. The decay in this transient regime is due to fast motion of spins that are initially free to evolve, that is, are initially facilitated by a sufficient number of up neighbors. The slow nonexponential late-time decay is due to spins that are initially blocked by the facilitation rule. Since the initial spin distribution is random we can use a mean-field argument to calculate the relaxation times of the initial transient exponential regime. At equilibrium the spin-up probability is given by $f=e^{-2 / k T} /\left(1+e^{-2 / k T}\right)$. Linear response around the equilibrium state $f+\delta e^{-t / \tau_{\beta}}$ yields $1 / \tau_{\beta}=p_{F}(f)\left(1+e^{-2 / k T}\right)$, where $p_{F}(f)$, the probability that a given site is facilitated, is given by the ratio

$$
\frac{\sum_{i=j}^{N} \frac{N !}{i !(N-i) !} f^{i}(1-f)^{N-i}}{\sum_{i=0}^{N} \frac{N !}{i !(N-i) !} f^{i}(1-f)^{N-i}} .
$$

Here $N$ is the total number of neighbors and $j$ is the minimum number of up neighbors needed for facilitation. The agreement between the calculated relaxation time $\tau_{\beta}(T)$ and 

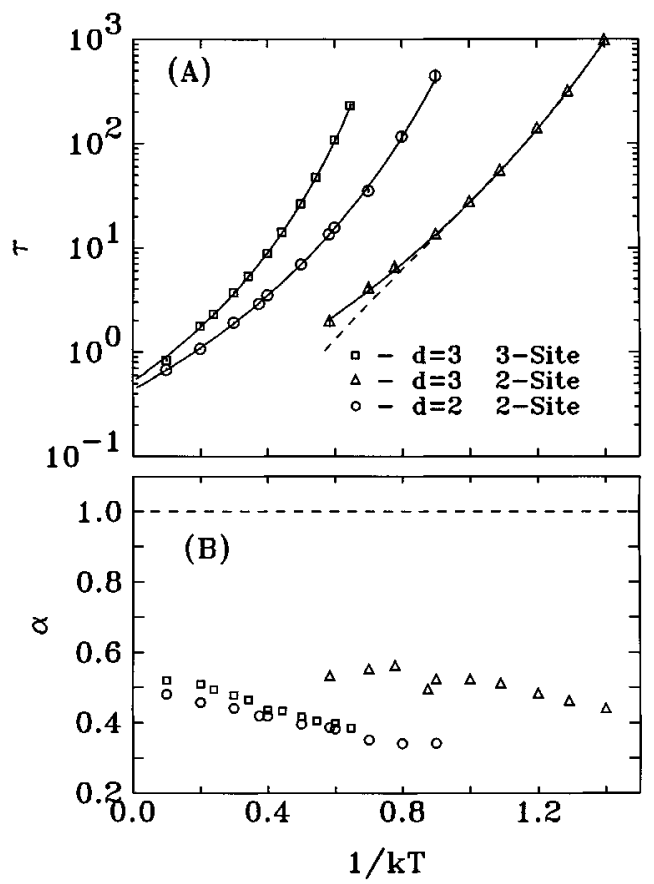

FIG. 4. Plot of the (a) relaxation times and (b) stretch powers as a function of inverse temperature $1 / k T$, for the three cases studied. Error bars (not shown) vary in size, from smaller than the displayed symbols to roughly twice the size of the symbols. A straight line in (a) corresponds to Arrhenius relaxation. The concave upward curves indicate divergence of the relaxation time at nonzero temperatures: the solid lines are the best fits using a Vogel-Fulcher function with the parameters given in Table I, while the dashed line is the best fit to a power law; the other fits are extremely poor. (b) indicates the possibility that the stretch power may be weakly dependent on temperature in the model.

the fits to the early-time simulation data are essentially exact and indicate Arrhenius behavior at low temperatures. To see this analytically we need only consider the limit of small $f$ (i.e., low temperatures), in which case the sums are dominated by their leading terms. The relaxation time is then

$$
\tau_{\beta} \sim \frac{e^{2 j / k T}}{\left(1+e^{-2 / k T}\right)^{j-1}} \approx e^{2 j / k T}
$$

which is an Arrhenius law. This is consistent with the interpretation of this short-time regime being analogous to the fast $\beta$-relaxation mechanism seen in experiment.

Let us return to the nonexponential late-time $\alpha$-relaxation regime and consider the fitted results for the relaxation times $\tau$ and stretch powers $\alpha$ (Fig. 4). Fig. 4(a) shows the temperature dependence of these relaxation times for the three models studied. A straight line implies Arrhenius relaxation and divergence of the relaxation time at $T=0$. All our results are concave upward, indicating divergence at a nonzero temperature. We fit these data to two possible forms for this divergence, a power law and the Vogel-Fulcher expression, and have summarized the results in Table I. These Vogel-Fulcher fits are shown in Fig. 4(a) with solid lines and a dashed line shows the best of the power-law fits; the other power-law fits are extremely poor and are not shown (note the $\chi^{2}$ values in Table I). Consid-
TABLE I. Results from fitting (a) Vogel-Fulcher $\left(\tau=c e^{A /\left(k T-k T_{0}\right)}\right)$ and (b) power-law $\left(\tau=A\left(k T-k T_{0}\right)^{-\gamma}\right)$ expressions to the relaxation time data shown in Fig. 4. The $\chi^{2}$ values are weighted by the uncertainty in the data.

\begin{tabular}{lcccc}
\hline \hline Model & $c$ & $A$ & $k T_{0}$ & $\chi^{2}$ \\
\hline \multicolumn{5}{c}{ (a) } \\
$d=2$ (two-site) & $0.43 \pm 0.02$ & $4.2 \pm 0.1$ & $0.50 \pm 0.03$ & 2.3 \\
$d=3$ (two-site) & $0.16 \pm 0.02$ & $3.6 \pm 0.1$ & $0.30 \pm 0.01$ & 2.1 \\
$d=3$ (three-site) & $0.51 \pm 0.02$ & $5.2 \pm 0.2$ & $0.68 \pm 0.03$ & 1.8 \\
\multicolumn{1}{c}{ Model } & $A$ & $\gamma$ & $k T_{0}$ & $\chi^{2}$ \\
\hline \multicolumn{5}{c}{ (b) } \\
$d=2$ (two-site) & $5.3 \pm 0.5$ & $1.3 \pm 0.1$ & $1.18 \pm 0.05$ & 118 \\
$d=3$ (two-site) & $1.8 \pm 0.1$ & $3.2 \pm 0.1$ & $0.57 \pm 0.01$ & 7.9 \\
$d=3$ (three-site) & $50 \pm 100$ & $3.0 \pm 2.0$ & $0.7 \pm 1.0$ & 264 \\
\hline \hline
\end{tabular}

ering all three models, it is clear that the Vogel-Fulcher expression provides a much better fit to the data than does the power law, showing the intimate relation between stretchedexponential time decay and the Vogel-Fulcher temperature dependence within this model. The relaxation times obtained from the integral of the curves gives essentially identical results. We have therefore shown that a microscopic, purely dynamical model can give rise to this important aspect of glassy phenomenology.

Figure 4(b) shows the temperature dependence of the stretch power $\alpha$, where $\alpha \sim 0.5$. Our results indicate a small systematic increase of $\alpha$ with temperature from about 0.35 to 0.55 with temperature. Up to now the consensus has been that the glassy regime is characterized by a single temperature-independent stretch exponent. However, we note that this weak temperature dependence of $\alpha$ is extracted from fits, so there may be systematic error in our data analysis of which we are not aware. Further study would be necessary to conclusively demonstrate a temperature dependence of $\alpha$. We also note that this feature could be difficult to observe experimentally.

The similarity between the two regimes of the facilitation model (fast exponential and slow stretch exponential) and the $\beta$ - and $\alpha$-relaxation mechanisms in true glasses is quite suggestive, although, in our simple model, we see no evidence of the elaborate effective temperature dependences and power-law decays seen in experimental $\beta$ behavior. The transient exponential $\beta$ mechanism is due to fast relaxation within a local, relatively free cage and the slower nonexponential $\alpha$ decay is due to slow diffusional relaxation of the trapped sites or particles. Thus our model for structural glasses reproduces both relaxation mechanisms present near the glass transition and provides a physically reasonable mechanism for both. We note that this physical argument has been previously discussed by Lewis [42].

Finally, we comment on a possible universal scaling of these relaxation data. At first, the simple form indicated by Fig. 4 would suggest that universal rescaling is possible. However, the fact that the early-time data follow $e^{-t / \tau_{\beta}(T)}$ precludes this possibility. Our data are consistent with two regimes, namely, the early-time exponential and the late-time stretched exponential. Naturally we cannot rule out other late-time scaling forms. However, for the late-time regimes 


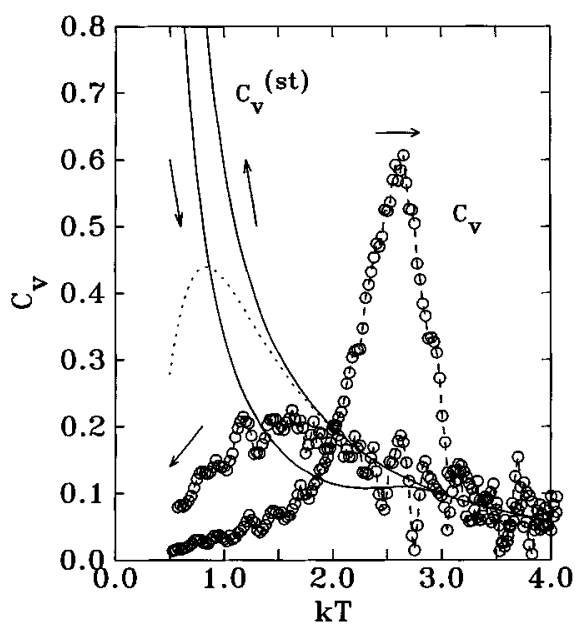

FIG. 5. Specific heat as a function of temperature $k T$ for a system heated from $T=0.45455$ to $T=6$ and subsequently cooled at a rate $|d(k T) / d t|=1 \times 10^{-5} k T / \mathrm{MCS}$. The arrows indicate the direction of the temperature change. The solid line gives the statistical specific heat $C_{v}^{(\text {st) }}$ defined in the text, while the open symbols linked by the dashed line give the experimentally relevant quantity $C_{v}=d\langle e\rangle / d(k T)$. The dotted line is the exact result in the absence of facilitation. The large heating peak corresponding to a glass transition is clearly evident, as is the breakdown of ergodicity for $k T \leqslant 2$ on cooling and $k T \leqslant 3$ on heating.

we studied our data are consistent with a stretched exponential and are inconsistent with a power law.

These results give confidence in the use of this simple model to investigate the glass transition. Hence we next investigated the thermal properties of model glass by quenching a system below the divergence temperature $T_{0}$ (indicated in Table I), relaxing it into a glassy state, and then slowly heating it, measuring the average system energy per spin $\langle e\rangle$ and fluctuations $\left\langle e^{2}\right\rangle-\langle e\rangle^{2} \equiv 1-\langle e\rangle^{2}$. The specific heat can be obtained two ways: by differentiating the energy per spin $C_{v}=d\langle e\rangle / d(k T)$ or by the statistical expression $C_{v}^{\text {(st) }}=N\left(\left\langle e^{2}\right\rangle-\langle e\rangle^{2}\right) /(k T)^{2}$. The latter expression can be incorrect when the system is nonergodic, while the former will reflect the thermal properties as they would be measured in an experiment. All these results can be contrasted with the exact results for this model in the absence of facilitation.

To study the ergodicity, we used the two-dimensional two-site facilitation model. Results for a typical heating and subsequent cooling run are shown in Fig. 5, contrasted with the exact Ising result (dotted line). This system was initially quenched from the all spin-up state to $k T=0.45455$ and allowed to relax for $8 \times 10^{6} \mathrm{MCS} / \mathrm{spin}$. It was then heated (and subsequently cooled), at a constant rate of $1 \times 10^{-5} \mathrm{kT} / \mathrm{MCS}$. This initial temperature is below the $T_{0} \approx 0.5$ of this system (see Table I) so the relaxation time of the prepared glassy state is extremely long: even after $8 \times 10^{6} \mathrm{MCS} / \mathrm{spin}$ the system was still evolving very slowly with time. However, relaxation was so slow that no noticeable change in properties would have occurred over the time scale of the heating-to-cooling run.

From the measurements of the specific heats, ergodicity, on the time scale of our simulation measurements, breaks down just below $k T=2.0$ for both heating and cooling runs. The heating run shows a large peak in the specific heat

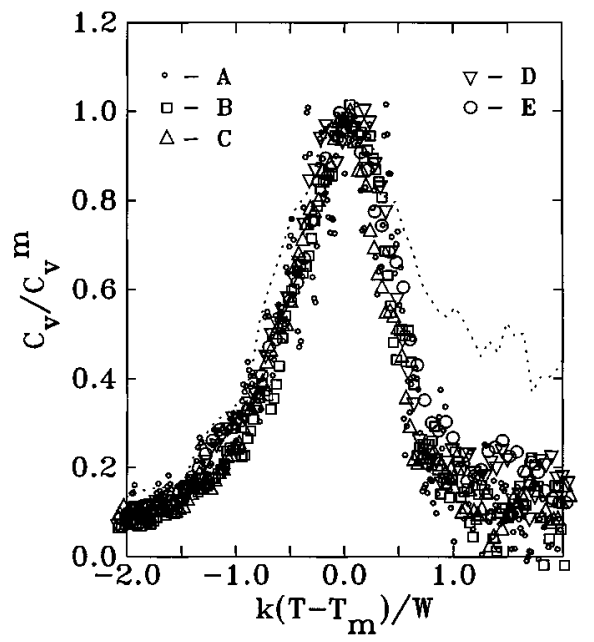

FIG. 6. Scaling plot for the glass transition. The data have been scaled according to $k\left(T-T_{m}\right) / W, C_{v} / C_{v}^{m}$, where $T_{m}$ is the position of the midpoint of the peak, $W$ is the full width at half maximum, and $C_{v}^{m}$ is the peak height. The heating rates corresponding to the different curves are (a) $5 \times 10^{-3} k T / \mathrm{MCS}$, (b) $1 \times 10^{-3} \mathrm{kT} / \mathrm{MCS}$, (c) $5 \times 10^{-4} \mathrm{kT} / \mathrm{MCS}$, (d) $2 \times 10^{-4} \mathrm{kT} / \mathrm{MCS}$, and (e) $1 \times 10^{-4} k T / M C S$. The dotted line (which does not scale) is the scaling result for systems heated at the very slow rate $5 \times 10^{-6} k T / \mathrm{MCS}$.

$C_{v}$, while the subsequent cooling run shows a much smaller and broader peak centered at a slightly lower temperature. If we reduce the scanning rate $d(k T) / d t$ the large peak narrows and moves to lower temperatures, while the broad cooling peak is largely unchanged (results not shown). This is also observed with real glasses. The calorimetric behavior of our model thus possesses much of the characteristic phenomenology of the thermally measured glass transition and the position of the maximum in the specific heat can be interpreted as the effective glass transition temperature $T_{g}$, which we will denote as $T_{m}$ below.

The quantity $C_{v}^{(\text {st) }}$ (solid line in Fig. 5) tells us about the microscopic ramifications of the broken ergodicity associated with this peak. Since this quantity diverges at low temperatures we see that $\left\langle e^{2}\right\rangle-\langle e\rangle^{2}$ does not go to zero as $T \rightarrow 0$. This means that fluctuations are quenched, on the time scale of the experiment, in keeping with dynamical arguments for the origin of glassy behavior.

Also of interest are the results of scaling the specific-heat data for calorimetric experiments performed at different heating rates. We analyzed such data using the standard scaling form, with results shown in Fig. 6. Provided the nonequilibrium peak is well displaced from the broad background peak of the equilibrium model (i.e., it is at sufficiently high temperatures) the data scale very well. This ceases to be the case (dotted line) when a large portion of the specific heat is due to the equilibrium background (dotted line). We note that one can ensure that these two regions are well separated by making the facilitation even more restrictive.

There has been interest in determining if, at asymptotically slow heating rates, the broad glass transition sharpens up in the manner of true first- or second-order transitions [17]. This is usually done by measuring the glass transition peak position and width, estimating their functional dependence over experimentally accessible heating and cooling 

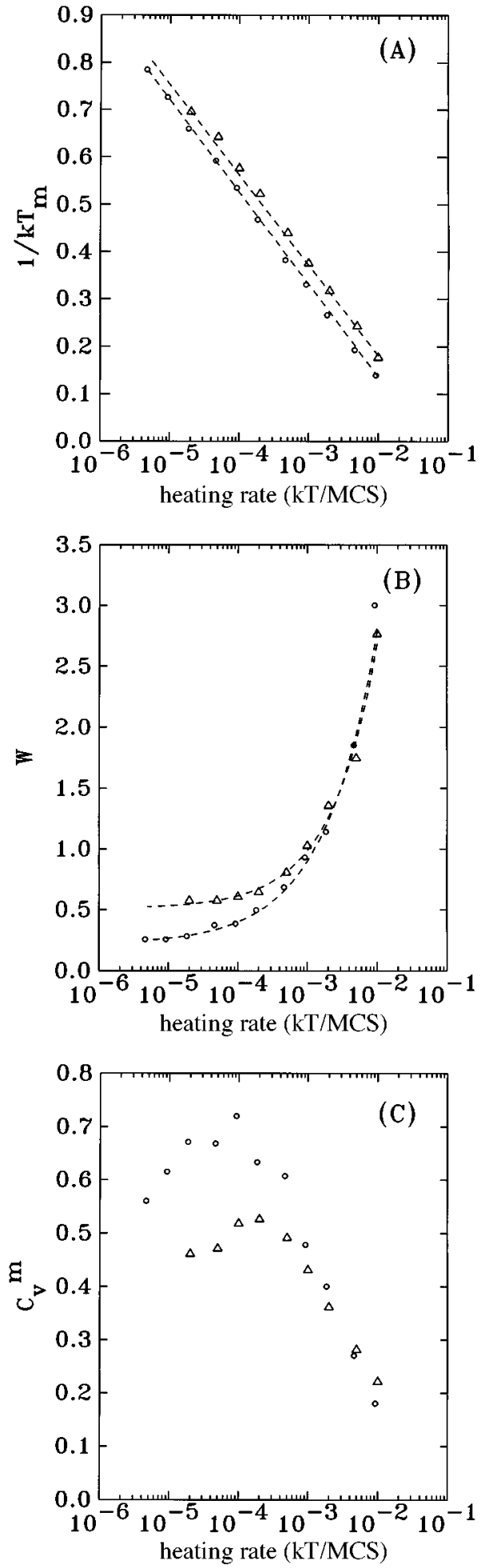

FIG. 7. Dependence of scaling parameters (a) $T_{m}$, (b) peak width $W$, and (c) specific-heat maximum $C_{v}^{m}$ on the heating rate. Results are shown for two initial states annealed for different lengths of time at $k T=0.45455: 8 \times 10^{6} \mathrm{MCS} /$ spin (circles) and $2 \times 10^{6} \mathrm{MCS} / \mathrm{spin}$ (triangles). The dashed lines in (a) are best fits to an Arrhenius law, while in (b) they correspond to power-law fits, as discussed in the text.

rates, and then extrapolating to slower rates. In our case this is equivalent to measuring the heating-rate dependence of the scaling parameters, which we have plotted in Fig. 7.

Figure 7(a) shows the rate dependence of the peak maxi- mum $T_{m}$ : the Arrhenius form is clearly evident. Similar Arrhenius behavior for the glass transition temperature is often seen in typical calorimetric experiments $[25,62]$ and implies that the effective activation energy arising from the dynamical frustration is independent of the heating rate. To check that this is a function only of the frustration mechanism and not of the annealed state we repeated the numerical simulations, starting with an initial state annealed for a much shorter time at $k T=0.45455\left(2 \times 10^{6} \mathrm{MCS} / \mathrm{spin}\right.$ as opposed to $\left.8 \times 10^{6} \mathrm{MCS} / \mathrm{spin}\right)$. The results, also shown in Fig. 7(a), show that the effective activation energy is largely independent of the initial state of the system.

Also of interest is the rate dependence of the peak width, plotted in Fig. 7(b). We have attempted to fit these data via power series in heating rate or by a power law with an additive constant $W=A+B t^{\alpha}$. The power law provides by far the better fit and yields a power-law growth exponent for the peak width of approximately 0.6. More importantly, this power law extrapolates to a finite-width transition at slow heating rates. Of course this extrapolation only makes sense for time scales shorter than the annealing time of the prepared low-temperature system. This also applies to real experiments.

In Fig. 7(c) we plot the specific-heat maximum as a function of heating rate. In general, the maximum increases with decreasing heating rate, until a critical rate is reached whereupon the maximum begins to decrease. The value of the maximum, as well as the crossover point between these two regimes, depends on the annealing time of the starting state. A longer annealing time yields larger specific-heat maxima and at the same time lowers the heating rate at which the crossover from increasing maxima to decreasing maxima is seen. We also examined the annealing time dependence by examining, at a fixed heating rate of $5 \times 10^{-4} \mathrm{kT} / \mathrm{MCS}$, the effective glass transition peak for system annealed for times between $4 \times 10^{5}$ and $8 \times 10^{6} \mathrm{MCS} /$ spin. We observe that the maximum in the specific heat $C_{v}^{m}$ and the temperature of this peak $T_{m}$ both increase with increasing annealing time, while the peak width $W$ decreases (data not shown). In addition, the integral over the peak estimated by the product $C_{v}^{m} W$ increases with aging. This is quite consistent with experimental observations of typical glasses. The numerical data are, however, too noisy for us to be able to estimate the functional form of these dependences.

Finaly, we examine the response of the glass state to an upward temperature jump. For this study we took configurations that had been annealed for $8 \times 10^{6} \mathrm{MCS} / \mathrm{spin}$ at $k T=0.45455$, instantaneously jumped the temperature to a higher value, and monitored the subsequent time evolution of the magnetization and the ensemble-averaged structure factor $S(q, t)$. Jumps were performed to temperatures between $k T=1.0$ and $k T=6.0$. Characteristic results are for magnetization and extreme- $q$ components of the structure factor are shown in Fig. 8 for a jump to $k T=4.0$, while Fig. 9 shows a three-dimensional topographic map of the evolution of the structure factor with time.

The results can be summarized as follows. As the glass state evolves towards equilibrium the structure factor initially develops a peak at $q=0$, which subsequently decays. Meanwhile, the structure factor at large $q$ evolves monotoni- 


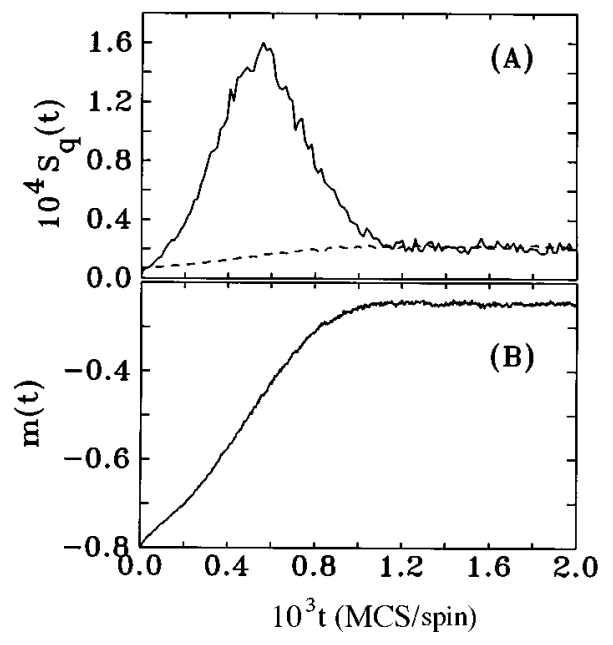

FIG. 8. Time dependence of the smallest- and largest- $q$ components of (a) the structure factor $S(q, t)$ and (b) the average magnetization for a temperature jump from $k T=0.45455$ to $k T=4.0$. The dashed line shows the result for large $q$, namely, $q=0.927$, in units of reciprocal lattice spacing. The solid line is for the smallest $q$, namely, $q=0.046247$.

cally towards the equilibrium value. The $q=0$ peak is clearly due to fluctuations in the magnetization: in fact, visualization of the spin configurations during the jump show the formation of domains of the fluctuating, high-temperature phase embedded within the still-frozen glass. The broad peak influences the structure factor out to approximately $q=0.35$, i.e., on the order of three inverse lattice spacings. Since each site in our model reflects an averaged domain in a real system, this implies quite large minimum domain sizes at the glassy peak.

The peak at $q=0$ is strongly reminiscent of structure factor evolution in spinodal decomposition when the order parameter is not conserved [63]. This is consistent with our model, which does not conserve magnetization. However, the dynamics of our model will be quite different from ordinary spinodal decomposition, since in the latter case the dynamics are driven by local free-energy gradients, while in our case the dynamics arise solely from the frustration.

We attempted to quantify this study by measuring the $q$ dependence of the relaxation times $\tau$ extracted from the
$S(q, t)$ curves. Unfortunately, our data are too noisy and do not extend to small enough $q$ for this to be feasible. We find that the rise in $S(q, t)$ at early times is best described by a power law, albeit with a power-law exponent that varies widely with $q$, but nonsystematically. Also, the drop in $S$ following the peak is described neither by a power law nor an exponential: the best description comes from a Wigner function $S \sim t^{\theta} e^{-t / \tau}$. Meanwhile, at high $q$ the structure factor grows almost linearly with time to the equilibrium value.

These results, however, are in qualitative agreement with the time-resolved x-ray diffraction study of Brauer [61]. In that temperature-jump study of glassy $\mathrm{Co}_{2} \mathrm{~B}$ and $\mathrm{Fe}_{76} \mathrm{~B}{ }_{24}$ Brauer found, prior to crystallization, a rapid rise in the structure factor at low $q$, but no equivalent rise at high $q$, similar to our results discussed above. In Brauer's case, however, this initial growth is exponential, allowing one to extract relaxation times as a function of $q$. Brauer found $\tau$ $\propto q^{2}$, consistent with the rise being due to density fluctuations. In addition, Brauer found the rapid rise in $S(q, t)$ only for $q<1.2 \times 10^{-2} \AA^{-1}$, corresponding to a length scale of $\sim 80 \AA$. This is consistent with our findings that the smallest length scale for the density fluctuations should be rather large.

We performed temperature jump experiments to various temperatures between $k T=1.0$ and 6.0. As with the glass transition data we attempted to scale these temperature jump results onto a universal curve. This proved unsuccessful for both the evolution of the structure factor evolution and the evolution of the average magnetization.

\section{DISCUSSION}

In this paper we presented and studied a facilitated kinetic Ising model for structural glasses. This model, a simplification of the model of Fredrickson and Andersen [6,7], considers a system of noninteracting Ising spins in a negative external field. Glasslike frustration, or facilitation, is introduced by allowing the spin at a given site to evolve via Metropolis dynamics only if it is surrounded by a sufficient number of up spins. If there are insufficient up neighbors the site is frozen and does not evolve. As the field increases (corresponding to decreasing temperature) the equilibrium state contains fewer and fewer up spins and more spins are frozen by the facilitation rule. This is analogous to saying that cold,

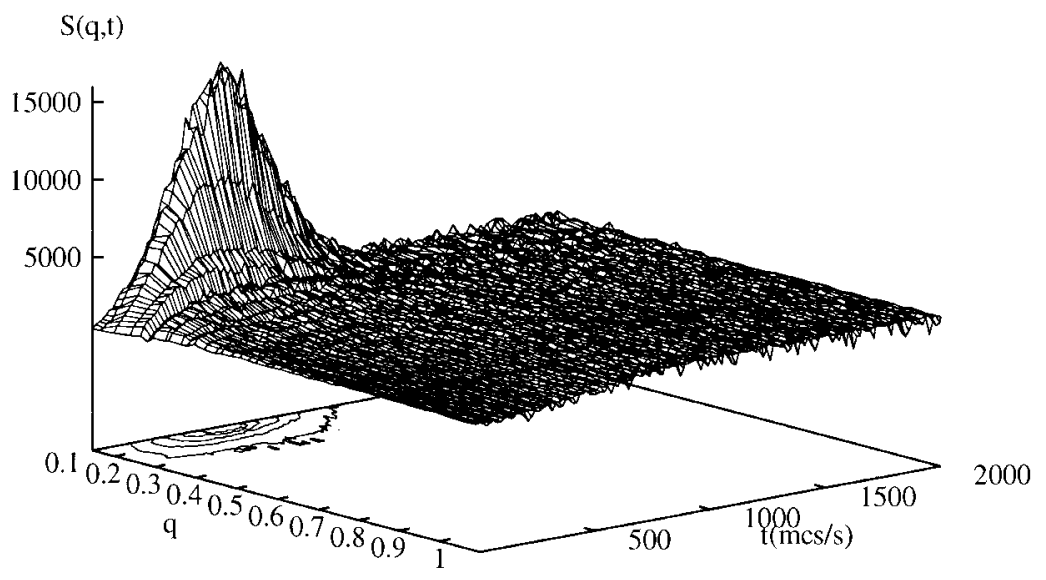

FIG. 9. Three-dimensional topographic plot of the structure factor $S(q, t)$ following a temperature jump from $k T=0.45455$ to $k T=4.0$. The contour plot in the $q$ - $t$ plane shows the structure factor peak at $q=0$. The structure factor at late time is flat, indicating systems at equilibrium. $S(q, 0)$, however, is slightly depressed at low $q$, reflecting the residual structure of the nonequilibrium glass. 
tightly packed regions of solid inhibit structural relaxation of their neighbors. At sufficiently low temperatures the facilitation requirement should frustrate relaxation completely, in which case the relaxation time should diverge and the system should become a glass.

The experimentally observed properties of glass-forming materials near but above the glass transition can be summarized as follows. There is an early-time power-law relaxation mechanism ( $\beta$ relaxation), which persists into the glass and has a temperature-dependent relaxation time that follows an Arrhenius law, for some regime of temperature and time. This relaxation mechanism is believed to be associated with fast local atomic rearrangements. At late times there is a second nonexponential relaxation mechanism ( $\alpha$ relaxation) that is often well described by a stretched exponential. The corresponding relaxation times are observed to diverge with temperature following a Vogel-Fulcher law $e^{A / k\left(T-T_{0}\right)}$, with a nonzero $T_{0}<T_{g}$. This is a diffusional relaxation mechanism and it is the cessation of this diffusion that allows a glass to form.

The results from the facilitation model are entirely consistent with these observations. We first note that there are two well-defined relaxation regimes. At short times the equilibrium autocorrelation function decays exponentially, with an associated relaxation time that follows an Arrhenius law. This relaxation is associated with local (fast) spin relaxation, but not spin diffusion. At later times the relaxation takes on a stretched-exponential form, with a stretch exponent $\alpha$ that depends no more than slightly on temperature and a prefactor that is temperature independent. In this case we measured the relaxation times and tested their dependence on temperature. In all cases studied we found their divergence best described via a Vogel-Fulcher law. This slow relaxation is due to the slow diffusion of up spins, which then facilitate the relaxation of otherwise frozen sites.

Given the success of the model reproducing these features of glasses, we turned to other typical experiments. We first modeled calorimetric experiments of glasses by heating a frozen model glass at a constant rate and measuring the evolved nonequilibrium heat. In an experiment the glass transition is marked by a prominent peak or jump in the nonequilibrium specific heat under these conditions. Our numerical results are consistent with these observations, showing a prominent peak in effective specific heat that can be attributed to a glass transition. This peak is entirely due to nonequilibrium behavior. As the heating rate is lowered this peak narrows and moves to lower temperatures. This is also consistent with experiments on structural glasses. In addition, our model indicates a universal scaling of the nonequilibrium specific-heat curves for different heating rates. To our knowledge, such scaling has not been attempted with experimental data.

Finally, we turned to temperature jump experiments and measured the evolution of the structure factor of a frozen glass following a jump to temperatures above $T_{g}$. At large $q$ the results show a monotonic increase of the structure factor towards the equilibrium value. The low- $q$ data, on the other hand, show the formation of a large peak at $q=0$, which then disappears as equilibrium is approached. As mentioned above, these results are qualitatively consistent with the time-resolved x-ray measurements of Brauer [61]. How- ever, our data are too poor to allow qualitative measurement of relaxation times.

The agreement between our model and experiment is not complete. For example, our model does not reproduce the complicated transient time and temperature dependence of the $\beta$-relaxation regime. Furthermore, within the $\alpha$-relaxation regime, our model indicates a temperatureindependent prefactor $c=O(1)$, different from the predictions of mode-coupling theory and from some experiments. Finally, the effective thermodynamics upon heating a model glass are only qualitatively similar to data on real glasses.

However, despite these drawbacks, the agreement between the numerical results of our model and the known phenomenology of glasses is quite remarkable, especially considering the extreme simplicity of our noninteracting kinetic Ising model. We therefore believe that the model contains the essential mechanism of glassy phenomena, namely, relaxation frustrated by local structure. In such models relaxation on long length scales (and hence long time scales) can only occur by the diffusion of up spins through the system. Meanwhile, the diffusion of these spins is frustrated by the paucity of up-spin neighbors. This hierarchical process serves to slow the diffusion and, for sufficiently few up spins, effectively stop it.

There are several remaining issues. First, we note that our model considers the facilitation energy barrier to be either zero (the site is facilitated) or infinity (the site cannot evolve). Thus the energy surface is filled with infinitely high (or zero-height) barriers separating different spin configurations. However, one could reasonably assume different energy barriers depending on the degree of facilitation. This would change significantly the shape of the energy surface, adding barriers of various heights. We note that the approach of Fredrickson and Andersen [6,7] includes such a modification to the energy surface. In their model the insufficiently facilitated sites never evolve (infinite barrier), while those that can evolve have their flipping probabilities weighted by the number of spin-up neighbors [59]. In this case the simulation results did not show the rich glasslike phenomenology we observe. In light of the success of our simpler model a reinvestigation of this more general approach would seem to be in order.

We have also ignored the role of the conservation law. In our treatment the magnetization was nonconserved. This is consistent with structural glasses, but may not be consistent with network glasses, where the number of network bonds is conserved. The conservation of this quantity, in the context of our model, may explain why network glasses make such strong glass-forming materials and also why network glasses show Arrhenius, not Vogel-Fulcher, divergences of their slow relaxation times. Simulation studies of variants of the facilitation model may answer some of these questions.

Finally, we point out the need for an analytic study. Since the facilitation model appears to encompass the relevant mechanism for glasses, studies of this model should shed light on the analytic properties of glassy relaxation. In addition, we suspect that the hydrodynamic approach, taken to the appropriate limit, will map onto a facilitation model. This might prove a useful way of explaining the origins of certain terms in the mode-coupling memory kernel. 


\section{ACKNOWLEDGMENTS}

We wish to thank Dr. K. Elder, Dr. R. Brüning, and Dr. L. Lewis for useful discussions and Professor D. Stauffer for useful comments on a preliminary version of this paper. This work was supported by the Natural Sciences and Engineering Research Council of Canada, les Fonds pour la Formation de Chercheurs et l'Aide à la Recherche de la Province du Québec.
[1] F. Spaepen and D. Turnbull, Annu. Rev. Phys. Chem. 35, 241 (1984).

[2] G.B. McKenna, in Comprehensive Polymer Science Vol 2: Polymer Properties, edited by C. Booth and C. Price (Pergamon, Oxford, 1989).

[3] J. Jäckle, Philos. Mag. B 56, 113 (1987).

[4] J. Adler, Physica A 171, 453 (1991) reviews the related topic of bootstrap percolation.

[5] H. Vogel, Phys. Z. 22, 645 (1921); G.S. Fulcher, J. Am. Ceram. Soc. 8, 339 (1925).

[6] G.H. Fredrickson and H.C. Andersen, Phys. Rev. Lett. 53, 1244 (1984); J. Chem. Phys. 83, 5822 (1985).

[7] G.H. Fredrickson and S.A. Brawer, J. Chem. Phys. 84, 3351 (1985).

[8] H. Hakanishi and H. Takano, Phys. Lett. A 115, 117 (1986).

[9] A preliminary account of this work has been published in a short paper: I. Graham, L. Piché, and M. Grant, J. Phys. Condens. Matter 5, L349 (1993).

[10] D. Turnbull and M.H. Cohen, J. Chem. Phys. 34, 120 (1961).

[11] M.H. Cohen and G.S. Grest, Phys. Rev. B 20, 1077 (1979); G.S. Grest and M.H. Cohen, ibid. 21, 4113 (1980).

[12] Gibbs and DiMarzio examined glass transitions in polymer systems using lattice statistics; in this case the free volume corresponds directly to sites in the lattice unoccupied by the chain segment. The minimum free volume is then equivalent to the point where the system entropy goes to zero. See, for example, J.H. Gibbs and E.A. DiMarzio, J. Chem. Phys. 28, 373 (1958) and Ref. [2].

[13] R. Brüning (private communication), C.T. Moynihan, S.M. Opalka, R. Mossadegh, S.N. Crichton, and A.J. Bruce, in Molecular Dynamics and Relaxation Phenomena in Glasses, edited by Th. Dorfmüller and G. Williams (Springer-Verlag, Berlin, 1987); H.W. Gronert, F. Gillessen, and D.M. Herlach, Mater. Sci. Eng. 97, 191 (1988); C.T. Moynihan, A.J. Easteal, J. Wilder, and J. Tucker, J. Phys. Chem. 78, 2673 (1974).

[14] A. Sahnoune, F. Massines, and L. Piché, J. Polymer Sci. (Phys. Ed.) 34, 341 (1996).

[15] Y.H. Jeong, S.R. Nagel, and S. Bhattacharya, Phys. Rev. B 34, 602 (1986).

[16] See Ref. [2] and references therein; A.J. Kovacs, J. Polym. Sci. 30, 131 (1958).

[17] R. Brüning and K. Samwer, Phys. Rev. B 46, 11318 (1992).

[18] E. Rössler, J. Chem. Phys. 92, 3275 (1990); Phys. Rev. Lett. 65, 1595 (1990).

[19] Y.H. Jeong, Phys. Rev. A 36, 766 (1987).

[20] N.J. Tao, G. Li, and H.Z. Cummins, Phys. Rev. Lett. 66, 1334 (1991).

[21] D.A. Pinnow, S.J. Candau, J.T. LaMaccia, and T.A. Litovitz, J. Acoust. Soc. Am. 43, 131 (1968).

[22] C. Demoulin, C.J. Montrose, and N. Ostrowsky, Phys. Rev. A 9, 1740 (1974).
[23] K. Schmidt-Rohr and H.W. Spiess, Phys. Rev. Lett. 66, 3020 (1991).

[24] D.W. McCall, D.C. Douglass, and D.R. Falcone, J. Chem. Phys. 50, 3839 (1969).

[25] P. Dixon, Phys. Rev. B 42, 8179 (1990).

[26] P.K. Dixon and S.R. Nagel, Phys. Rev. Lett. 61, 341 (1988).

[27] N.O. Birge and S.R. Nagel, Phys. Rev. Lett. 54, 2674 (1985); N.O. Birge, Phys. Rev. B 34, 1631 (1986).

[28] A. Schönhals, F. Kremer, and E. Schlosser, Phys. Rev. Lett. 67, 999 (1991)

[29] P.K. Dixon, L. Wu, S.R. Nagel, B.D. Williams, and J.P. Carini, Phys. Rev. Lett. 65, 1108 (1990).

[30] G.P. Johari and M. Goldstein, J. Chem. Phys. 55, 4245 (1971); 53, 2372 (1970).

[31] U. Buchenau, M. Prager, N. Nüker, A.J. Dianoux, N. Ahmad, and W.A. Phillips, Phys. Rev. B 34, 5665 (1986).

[32] F. Mezei, W. Knaak, and B. Farago, Phys. Rev. Lett. 58, 571 (1987).

[33] J. Fujara and W. Petry, Europhys. Lett. 4, 921 (1987).

[34] W. Knaak, F. Mezei, and B. Farago, Europhys. Lett. 7, 529 (1988).

[35] W.A. Phillips, U. Buchenau, N. Nücker, A.-J. Dianoux, and W. Petry, Phys. Rev. Lett. 63, 2381 (1989).

[36] B. Frick, B. Farago, and D. Richter, Phys. Rev. Lett. 64, 2921 (1990).

[37] D. Richter, B. Frick, and B. Farago, Phys. Rev. Lett. 61, 2465 (1988).

[38] M. Kiebel, E. Bartsch, O. Debus, F. Fujara, W. Petry, and H. Sillescu, Phys. Rev. B 45, 10301 (1992); E. Bartsch, O. Debus, F. Fujara, M. Kiebel, W. Petry, H. Sillescu, and J.H. Magill, Physica A 180\&181, 808 (1992); W. Petry, E. Bartsch, F. Fujara, M. Kiebel, H. Sillescu, and B. Farago, Z. Phys. B 83, 175 (1991).

[39] Reviews of molecular-dynamics studies of glasses are J.-L. Barrat and M.L. Klein, Annu. Rev. Phys. Chem. 42, 23 (1991); C.A. Angell, J.H.R. Clarke, and L.V. Woodcock, Adv. Chem. Phys. 48, 397 (1981).

[40] J.J. Ullo and S. Yip, Phys. Rev. Lett. 54, 1509 (1985).

[41] G.F. Signorini, J.-L. Barrat, and M.L. Klein, J. Chem. Phys. 92, 1294 (1990).

[42] L.J. Lewis, Phys. Rev. B 44, 4245 (1991); L.J. Lewis and G. Wahnström, Solid State Commun. 86, 295 (1993); Phys. Rev. E 50, 3865 (1994).

[43] P. Taborek, R.N. Kleiman, and D.J. Bishop, Phys. Rev. B 34, 1835 (1986).

[44] W. Götze, in Liquids, Freezing and the Glass Transition, edited by D. Levesque, J.P. Hansen, and J. Zinn-Justin (Elsevier, New York, 1991).

[45] B. Kim and G.F. Mazenko, Phys. Rev. B 45, 2393 (1992).

[46] D.W. Davidson and R.H. Cole, J. Chem. Phys. 19, 1484 (1951). 
[47] G. Pastore, B. Bernu, J.P. Hansen, and Y. Hiwatari, Phys. Rev. A 38, 454 (1988).

[48] J.-L. Barrat, J.-N. Roux, and J.-P. Hansen, Chem. Phys. 149, 197 (1990).

[49] Y. Hiwatari and H. Miyagawa, J. Non-Cryst. Solids 117/118, 862 (1990).

[50] F. Stickel, F.W. Fischer, and R. Richert, J. Chem. Phys. 102, 6251 (1995); 104, 2043 (1996), and references therein.

[51] Various models for slow relaxation in glassy systems are summarized by R.G. Palmer, in Cooperative Dynamics in Complex Physical Systems, edited by H. Takayama, Springer Series in Synergetics Vol. 43 (Springer-Verlag, Berlin, 1989).

[52] R.G. Palmer, D.L. Stein, E. Abrahams, and P.W. Anderson, Phys. Rev. Lett. 53, 958 (1984).

[53] D. Leutheusser, Phys. Rev. A 29, 2765 (1984).

[54] U. Bentzelius, W. Götze, and A. Sjölander, J. Phys. C 17, 5915 (1984); W. Götze, Z. Phys. B 60, 195 (1985); H. De Raedt and W. Götze, J. Phys. C 19, 2607 (1986); W. Götze and L. Sjögren, Z. Phys. B 65, 415 (1987); W. Götze, Z. Phys. Chem. 156, 3 (1988).

[55] S.P. Das and G.F. Mazenko, Phys. Rev. A 34, 2265 (1986); S.P. Das, ibid. 36, 211 (1987); B. Kim and G.F. Mazenko, Adv. Chem. Phys. 78, 129 (1990).

[56] W. Götze and L. Sjögren, Z. Phys. B 65, 415 (1987).

[57] W. Götze, Z. Phys. Chem. 156, 3 (1988).
[58] D. Walton, Phys. Rev. Lett. 65, 1599 (1990); Phys. Rev. B 44, 11655 (1991).

[59] In the Fredrickson-Andersen model the facilitation function depends on the actual number of neighboring up spins $m_{j}$. For example, their two-site rule is $f \sim \frac{1}{2} m_{j}\left(m_{j}-1\right)$, so that the flipping probability, when two or more neighbors have up spins, is weighted by the number of up-spin neighbors. By choosing $f=\{0,1\}$ we preserve the essence of the model: that spins with insufficient up-spin neighbors cannot evolve and have merely simplified the microscopic dynamics for those that do evolve. The advantage is that the extrapolation to a zero-site rule yields the kinetic Ising model, which is not the case for the original Fredrickson-Andersen model.

[60] I.S. Graham and M. Grant, J. Phys. A 25, L1195 (1992); C. Roland and M. Grant, Phys. Rev. B 39, 11971 (1989); G. Bhanot, D. Duke, and R. Salvador, J. Stat. Phys. 44, 985 (1986).

[61] S. Brauer, Ph.D. thesis, McGill University, 1992 (unpublished).

[62] J. Jäckle, Rep. Prog. Phys. 49, 171 (1986); R. Lück, Q. Jiang, and B. Predel, J. Non-Cryst. Solids 117/118, 911 (1990); C.T. Moynihan, A.J. Easteal, J. Wilder, and J. Tucker, J. Phys. Chem. 78, 2673 (1974).

[63] See, for example, J.D. Gunton, M. San Miguel, and P.S. Sahni, in Phase Transitions and Critical Phenomena, edited by C. Domb and J.L. Lebowitz (Academic, London, 1983), Vol. 8. 